\title{
Enhanced ferroelectric and ferromagnetic properties in lead-free multilayer composite films based on ferroelectric $\left(\mathrm{Bi}_{0.5} \mathrm{Na}_{0.5}\right)_{0.945} \mathrm{Ba}_{0.055} \mathrm{TiO}_{3}$ and multiferroic $\mathrm{BiFeO}_{3}$
}

\author{
A. Perez-Rivero, J. Ricote, I. Bretos, M. García-Hernández, M. L. Calzada, and R. Jiménez \\ Instituto de Ciencia de Materiales de Madrid, CSIC, Cantoblanco, 28049 Madrid, Spain
}

(Received 19 November 2014; accepted 2 February 2015; published online 10 February 2015)

\begin{abstract}
The study of the functional properties of the multilayer composite films of ferroelectric $\left(\mathrm{Bi}_{0.5} \mathrm{Na}_{0.5}\right)_{0.945} \mathrm{Ba}_{0.055} \mathrm{TiO}_{3}(\mathrm{BNBT})$ and multiferroic $\mathrm{BiFeO}_{3}(\mathrm{BF})$ obtained by chemical solution deposition has been performed in this work. First, it has been observed a significant improvement of the remnant polarization of the multilayer composite with respect to the single phase film of BNBT: $\mathrm{P}_{\mathrm{r}}=32 \mu \mathrm{C} \times \mathrm{cm}^{-2}$, which is comparable with the values reported for bulk BNBT. This is a consequence of the stabilization of the ferroelectric domains of BNBT, although the relaxor character is still present. The leakage currents, which prevent the full exploitation of the properties of BF films, are reduced thanks to the combination with insulator BNBT layers. Besides, the magnetic behavior shows values of remnant magnetization at room temperature that makes this multilayer composites promising for multiferroic applications. (c) 2015 AIP Publishing LLC.

[http://dx.doi.org/10.1063/1.4908069]
\end{abstract}

\section{INTRODUCTION}

Ferroelectric thin films are being used nowadays integrated in a variety of microdevices, like microwave electronics, non-volatile memories, and piezoelectric microsensors and actuators. ${ }^{1}$ The stacking of layers of different ferroelectrics in a multilayer composite (MLC) configuration has proven successful for the improvement of the dielectric and ferroelectric properties of single phase films. ${ }^{2}$ Some superlattices and multilayers displayed enhanced polarization thanks to the interactions between the layers. ${ }^{3,4}$ This is especially important for compositions like relaxorferroelectric, giant piezoelectric $(1-\mathrm{x}) \mathrm{Pb}\left(\mathrm{Mg}_{1 / 3} \mathrm{Nb}_{2 / 3}\right) \mathrm{O}_{3^{-}}$ $\mathrm{xPbTiO}_{3}$ (PMNT) solid solution close to the morphotropic phase boundary (MPB). This perovskite shows reduced remnant polarization and, therefore, reduced piezoelectric coefficients in thin film form due to a grain size effect. ${ }^{5}$ This is an important limiting factor for their use integrated in microdevices. The effect on the polarization of the PMNT layers by neighboring poled ferroelectric layers in a multilayer composite film results in enhanced remnant polarization. ${ }^{6}$

Most of single phase and multilayered films contain $\mathrm{Pb}(\mathrm{Zr}, \mathrm{Ti}) \mathrm{O}_{3}$ (PZT) due to its excellent ferroelectric properties. It presents an enhancement of its properties near the MPB between the rhombohedral and tetragonal phases of this solid solution. ${ }^{7}$ However, the strict environmental regulations that are being enforced worldwide prohibit the use of lead-based materials, which has triggered the search of alternative ferroelectric alternative lead-free materials. ${ }^{8}$ Among other compositions, the solid solution $(1-\mathrm{x})\left(\mathrm{Bi}_{0.5} \mathrm{Na}_{0.5}\right)$ $\mathrm{TiO}_{3}-\mathrm{XBaTiO}_{3}$ (BNBT) has been presented as a very promising candidate. ${ }^{9}$ It shows large piezoelectric coefficients for compositions in the vicinity of a MPB. ${ }^{9,10}$ Recently, it has been reported relaxor-ferroelectric character in the properties of this solid solution for compositions close to the MPB. ${ }^{11}$ In thin film form significant changes are observed respect to the bulk. $^{12,13}$ The room temperature phase at the MPB in films shows a strong relaxor character, which results in a drastic reduction of the remnant polarization. This is similar to the behavior of relaxor ferroelectric PMNT thin films mentioned above, ${ }^{5}$ which has been attributed to the stabilization of a relaxor state associated to the reduction of the grain size. ${ }^{14}$ The reduction of the remnant polarization of the BNBT films affects all the ferroelectricity-related functional properties and, therefore, restricts their application in microdevices.

In order to improve the properties of BNBT based films, we analyze in this work the combination of this material with a large ferroelectric polarization in a 2-2 biphasic multilayer composite film, similarly to that reported in Ref. 6. One of the best lead-free candidates is the perovskite of bismuth ferrite, $\mathrm{BiFeO}_{3}(\mathrm{BF}){ }^{15}$ It is perhaps the most widely studied multiferroic material, in part due to its large ferroelectric and magnetic ordering temperatures. BF in thin film form presents enhanced polarization and magnetization values, ${ }^{16}$ although the low film resistivity is a problem to access this polarization values at room temperature. This can be solved by the application of an electricconditioning treatment, ${ }^{17}$ and thus BF layers show $\mathrm{P}_{\mathrm{r}} \approx 60 \mu \mathrm{C} \times \mathrm{cm}^{-2}$ at room temperature. ${ }^{18}$ These values make them excellent candidates to be used in a multilayer configuration to induce polarization in the BNBT layers. Moreover, we can also take advantage of the good ferroelectric and magnetization values of the BF layers for multiferroic applications in the proposed 2-2 biphasic multilayer composites, as the BNBT may act as a point defect buffer for the BF, reducing their leakage currents. Ferroelectric and magnetic properties of these multilayer composite films will be analyzed, and the mechanisms for the enhancement of the effective remnant polarization of the composite thin films discussed. 


\section{EXPERIMENTAL PROCEDURE}

Thin films were prepared by chemical solution deposition (CSD). BNBT layers were obtained from a precursor sol of $\left(\mathrm{Bi}_{0.5} \mathrm{Na}_{0.5}\right) \mathrm{TiO}_{3}$ and a precursor solution of $\mathrm{BaTiO}_{3}$, which were prepared following a solution method reported elsewhere. ${ }^{12,19,20} \mathrm{BF}$ layers were deposited from solutions according to the procedure reported in Ref. 18. Successive layers of the diluted precursor solutions were spin-coated at $2000 \mathrm{rpm}$ for $45 \mathrm{~s}$ onto (111) $\mathrm{Pt} / \mathrm{TiO}_{2} / \mathrm{SiO}_{2} /(100) \mathrm{Si}$ substrates and dried at $350{ }^{\circ} \mathrm{C}$ for $60 \mathrm{~s}$ in a hot plate. As-deposited amorphous layers were then crystallized by rapid thermal processing (RTP, JetStar 100T JIPELEC) with a heating rate of $\sim 30^{\circ} \mathrm{C} / \mathrm{s}$. BNBT layers were crystallized in oxygen at $650{ }^{\circ} \mathrm{C}$ for $60 \mathrm{~s}$ and $\mathrm{BF}$ layers in air at $500^{\circ} \mathrm{C}$ for $60 \mathrm{~s}$, according to the optimized processing parameters reported for each type of layer. ${ }^{12,18}$ The MLC films were fabricated by the successive deposition, drying, and crystallization of alternating BNBT and BF layers. For the MLC-BNBT film, first the deposition of 3 coatings of $\mathrm{BF}$ solution produce the bottom BF layer, on top of which we performed the deposition of 6 coatings of BNBT solution, to finish with a top BF layer prepared like the bottom $\mathrm{BF}$ one. In the case of the MLC-BF film, we started with 3 coatings of BNBT, followed by 6 of BF and finally 3 of BNBT. By this way, we obtained two multilayer composite films with two different configurations: $\mathrm{BF} / \mathrm{BNBT} / \mathrm{BF}$ for MLC-BNBT and BNBT/BF/BNBT for MLC-BF, but with the same volume fractions: $V_{B N B T}$ $=0.75$ and $\mathrm{V}_{\mathrm{BF}}=0.25$. Single phase films of both compositions were also prepared for the sake of comparison.

The study of the crystalline phases was carried out by X-ray diffraction using a D8-Bruker X-ray powder diffractometer (Bruker AXS Inc., Madison, WI), with a $\mathrm{Cu}$ anode and Bragg-Brentano geometry. The surface and crosssection images of the multilayers composite films were obtained by field-emission gun scanning electron microscope (FEG-SEM; Nova Nanosem 230 FEI Company equipment, Hillsboro, OR). Through this technique, it was possible to estimate the total thickness of both multilayer composite films, which was used for the calculation of the physical parameters in the paper.

For the functional electrical characterization of the different samples, top electrodes of $\mathrm{Pt} / \mathrm{Au}$ with $200 \mu \mathrm{m}$ of diameter approximately were deposited on the film surfaces by D.C. sputtering through a shadow mask. The ferroelectric characterization was carried out by the measurement of P-E hysteresis loops, which were performed in a home built system, based on a virtual ground circuit. ${ }^{21}$ In this system, a HP8116A pulse generator was used to apply sinusoidal voltage waves at a frequency of $1 \mathrm{kHz}$ and the loops were traced by a Tektronix TDS520 oscilloscope. This equipment coupled to a home built cryostat equipped with micro-manipulators allowed the measurement of the hysteresis loops from $100 \mathrm{~K}$ to $400 \mathrm{~K}$ in thin films. The non-ferroelectric switching contributions from the loops were eliminated by a fitting procedure based on a model that simulates the linear part of these contributions by introducing the capacitance and resistance of the films. ${ }^{22}$ Non-linear contributions, leakage currents, are fitted by a model that considers the electrical current proportional to $\mathrm{V}^{2} \cdot{ }^{23}$ The measurements of the remnant hysteresis loops, polarization retention, and leakage currents vs. electric field were carried out using commercial RADIANT Precision Premier II equipment. In this case, triangular voltage waves were used, with a delay time of $1 \mathrm{~s}$ between the conditioning and measuring voltages. The capacitance vs. voltage curves to obtain the low frequency coercive fields were measured in an Impedance Gain-Phase Analyzer HP 4194 A. These measurements were performed applying one wave of $5 \mathrm{kHz}$, biasing the capacitor with changing bias in a staircase way.

Magnetic field (M-H) hysteresis loops at 4.2 and $300 \mathrm{~K}$ were measured in the films, using a superconducting quantum interface device magnetometer from Quantum Design, with a 5-T coil. The magnetic field was applied parallel to the film surface, and the diamagnetic contributions from the substrate were eliminated by fitting the high field data to a straight line with a negative slope.

\section{RESULTS AND DISCUSSION}

The phases present in the two multilayer composite films were studied by X-ray diffraction, as shown in Figure 1, and compared with single phase films of BNBT and BF. It can be observed that both multilayer composites contain the desired crystalline phases for the layers: MPB $\left(\mathrm{Bi}_{0.5} \mathrm{Na}_{0.5}\right)_{0.945}$ $\mathrm{Ba}_{0.055} \mathrm{TiO}_{3}$ and $\mathrm{BiFeO}_{3}$. It must be pointed out that only a small peak associated to a secondary phase appears in the $\mathrm{X}$-ray patterns, coming from the $\mathrm{BiFeO}_{3}$ layers. It is close to $2 \theta \sim 29.9^{\circ}$, associated to the iron-rich $\mathrm{Bi}_{2} \mathrm{Fe}_{4} \mathrm{O}_{9}$ secondary phase. $^{18}$

The fact that only crystalline phases coming from the individual layers are present indicates that no significant interdiffusion between the alternating BNBT and BF layers is produced. As the diffraction peaks of these two phases are too close, details of the patterns are shown in Figures 1(b) and 1(c): experimental data (solid lines) with their corresponding deconvolution into the diffraction peaks from the BNBT and BF layers (dashed lines). Deconvolution of the experimental diffraction peaks was carried out by using two pseudo-Voigt functions. It must be noted that the MPB $\left(\mathrm{Bi}_{0.5} \mathrm{Na}_{0.5}\right)_{0.945} \mathrm{Ba}_{0.055} \mathrm{TiO}_{3}$ phase contains both rhombohedral and tetragonal crystalline phases, ${ }^{12}$ and deconvolution of the diffraction peaks is needed to show their respective contributions. ${ }^{24}$ For the purposes of this paper, we have not considered this mixture of phases, and we have indexed the reflections corresponding to BNBT on the basis of a pseudocubic (p) unit cell.

A columnar-like growth was observed in the SEM micrographs of cross sections of both multilayer composite films (Figures 2(a) and 2(b)). The measured film thickness is very similar: $470 \pm 20 \mathrm{~nm}$ and $500 \pm 20 \mathrm{~nm}$ for the MLC-BF and MLC-BNBT, respectively. From the number of solution depositions, we can estimate the thickness of the BNBT and BF layers; they are superimposed in the micrographs to show the approximate position of the layer boundaries. It can be seen that there are some observable traces of these boundaries in some regions, which indicates the different microstructure of both types of layers. While BF layers tend to have small pores (see surface image of the top BF layer in 

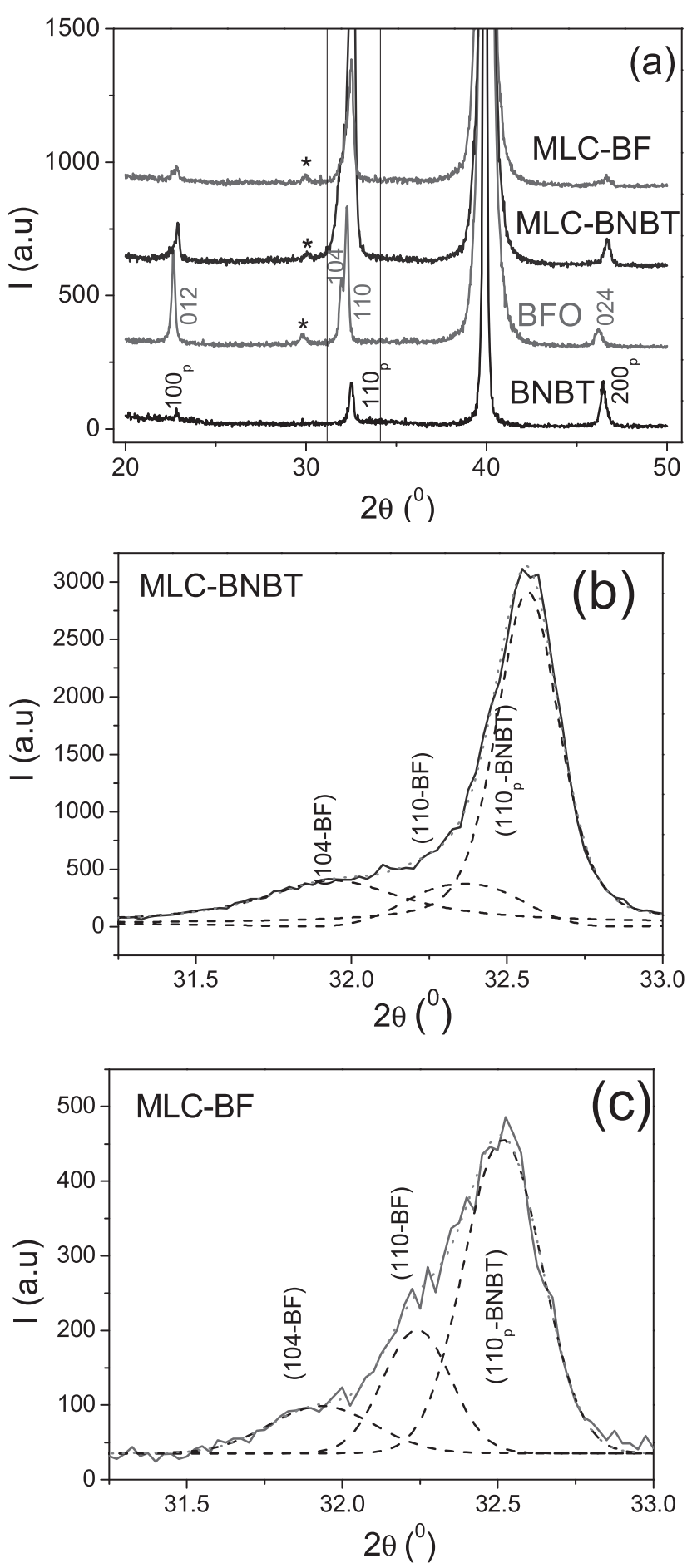

FIG. 1. (a) X-ray diffraction patterns of the single phase $\mathrm{BiFeO}_{3}$ and $\mathrm{BNBT}$ films compared with the multilayer composite films MLC-BNBT and MLC$\mathrm{BF}\left(* \mathrm{Bi}_{2} \mathrm{Fe}_{4} \mathrm{O}_{9}\right)$. (b) and (c) show the deconvolution of diffraction peaks for both MLCs.

the MLC-BNBT film of Figure 2(c)), BNBT layers present columns that are more separated, with larger voids between them, as it can be seen in Figure 2(d) where the surfaces of the top BNBT of the MLC-BF film is shown. This characteristic has been reported before for similar single phase BNBT films. ${ }^{12}$ This difference between the two types of layers made the columns rather irregular, which reduces the risk of short circuits between top and bottom electrodes through the column boundaries. Regarding the grain size, it can be seen that there are not significant differences, with the column width values around $100 \mathrm{~nm}$ for both multilayer composite films.

Corrected P-E hysteresis loops for both multilayer composite films are shown in Figure 3, measured at $150 \mathrm{~K}$ and $300 \mathrm{~K}$. The insets of this figure show the corresponding experimental current vs. electric field loops, from which we calculate the P-E loops. It is noticeable that, although it can be seen a small increase with the temperature, leakage currents are strongly reduced in MLC films in comparison with those reported for the single phase BF films. ${ }^{18}$ Although both composites contain the same volume ratio of BNBT and $\mathrm{BF}$ layers, the saturation and remnant polarization values differ: MLC-BNBT film show at $300 \mathrm{~K} \mathrm{P}_{\mathrm{s}}=43.6 \mu \mathrm{C} \times \mathrm{cm}^{-2}$ and $\mathrm{P}_{\mathrm{r}}=32.4 \mu \mathrm{C} \times \mathrm{cm}^{-2}$, while values for MLC-BF film are $\mathrm{P}_{\mathrm{s}}=40.3 \mu \mathrm{C} \times \mathrm{cm}^{-2}$ and $\mathrm{P}_{\mathrm{r}}=23.5 \mu \mathrm{C} \times \mathrm{cm}^{-2}$.

We can calculate the expected values for the remnant polarization of the MLC $\left(\mathrm{P}_{\mathrm{r}}^{\mathrm{MLC}}\right)$ from the values of the polarization of the individual layers $\left(\mathrm{P}_{\mathrm{r}}{ }^{\mathrm{BNBT}}\right.$ and $\left.\mathrm{P}_{\mathrm{r}}{ }^{\mathrm{BF}}\right)$ with the following equation that considers a linear additive effect of properties in the multilayer structure:

$$
\mathrm{P}_{\mathrm{r}}^{\mathrm{MLC}}=\mathrm{V}_{\mathrm{BNBT}} \mathrm{P}_{\mathrm{r}}^{\mathrm{BNBT}}+\mathrm{V}_{\mathrm{BF}} \mathrm{P}_{\mathrm{r}}^{\mathrm{BF}}
$$

being $\mathrm{V}$ the volume fraction of the phases $\left(\mathrm{V}_{\mathrm{BNBT}}=0.75\right.$, $\left.\mathrm{V}_{\mathrm{BF}}=0.25\right)$. Taking the remnant polarization values for single phase $\mathrm{BNBT}\left(\mathrm{P}_{\mathrm{r}}^{\mathrm{BNBT}}=12.3 \mu \mathrm{C} \times \mathrm{cm}^{-2}\right.$ (Ref. 12)) and $\mathrm{BF}$ films $\left(\mathrm{P}_{\mathrm{r}}^{\mathrm{BF}}=61.1 \mu \mathrm{C} \times \mathrm{cm}^{-2}\right.$ (Ref. 18)), we obtain an expected remnant polarization for the MLC films studied in this work: $\mathrm{P}_{\mathrm{r}}{ }^{\mathrm{MLC}}=24.5 \mu \mathrm{C} \times \mathrm{cm}^{-2}$. This simple equation is valid for high enough applied electric field and can be considered as a maximum expected value for the remnant polarization in the composite. Note that from the calculations using Eq. (1), an error in the estimation of the volume fraction of the layers of $10 \%$ produces a variation in the remnant polarization of $\pm 2 \mu \mathrm{C} \times \mathrm{cm}^{-2}$, which is lower than the observed change.

From this result, we can conclude that in the case of MLC-BF the remnant polarization value obtained $\left(\mathrm{P}_{\mathrm{r}}=23.5 \mu \mathrm{C} \times \mathrm{cm}^{-2}\right)$ corresponds to a simple addition of the properties in the composite film, and thus the applied field is large enough to produce the maximum expected remnant polarization. However, the larger value obtained for the MLC-BNBT $\left(\mathrm{P}_{\mathrm{r}}=32.4 \mu \mathrm{C} \times \mathrm{cm}^{-2}\right)$ indicates that other contributions must be considered. The increment of the polarization is here of $33 \%$. It should be borne in mind that the differences between the two MLC films are the ordering sequence of the layers and their thickness. In the MLCBNBT, the BNBT layer $(380 \mathrm{~nm})$ is sandwiched between two BF layers $(60 \mathrm{~nm})$, while in the MLC-BF film, the BF layer $(120 \mathrm{~nm})$ is between two BNBT layers $(175 \mathrm{~nm})$. Thickness may affect the polarization values of the layers due to a size effect. From the SEM images, it can be concluded that the grain size is not much influenced by the different thickness of the layers (see Figure 2). Therefore, a size effect should not be significant, although it cannot be discarded completely. To verify the effect of the ordering sequence of the layers on the enhanced remnant polarization, the electrical behavior of a 2-2 composite made out of two 

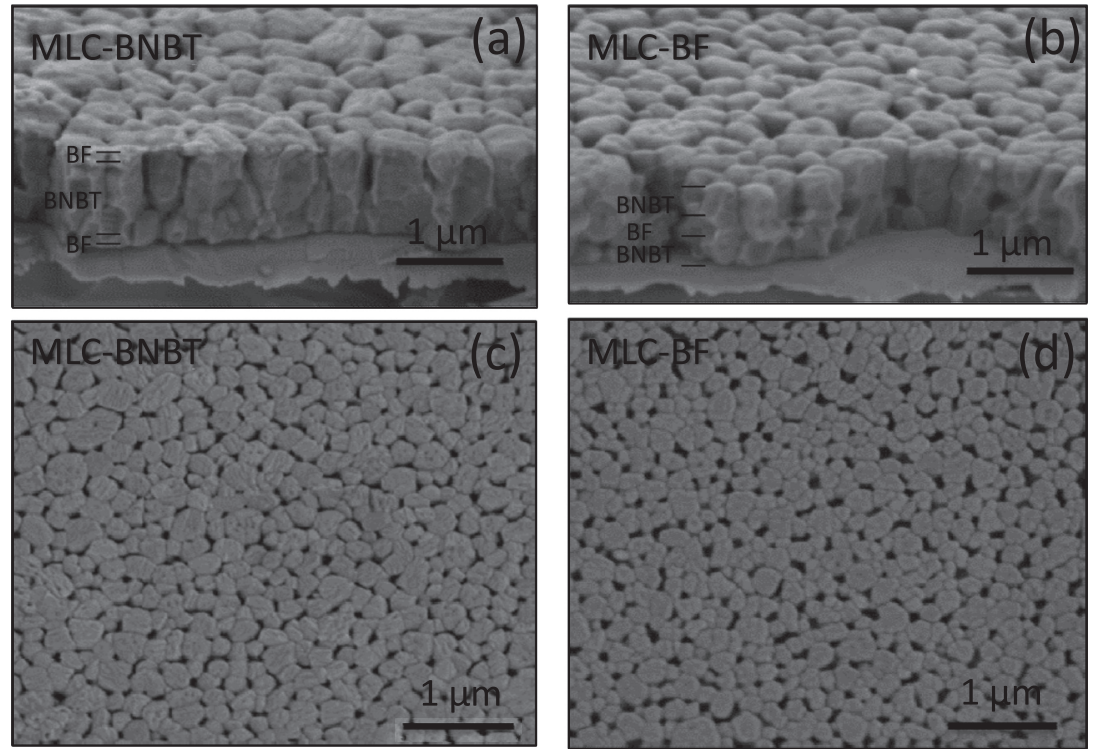

FIG. 2. SEM micrographs of: (a) and (b) cross sections of MLC-BNBT and MLC-BF film, respectively; (c) and (d) top surfaces of MLC-BNBT and MLCBF film, respectively. ferroelectric layers (bilayer) with electrodes has been calculated. In Figure 4, a schematic diagram of the proposed composite and the equivalent circuit are shown, together with the calculated P-E hysteresis loops, which are compared to the
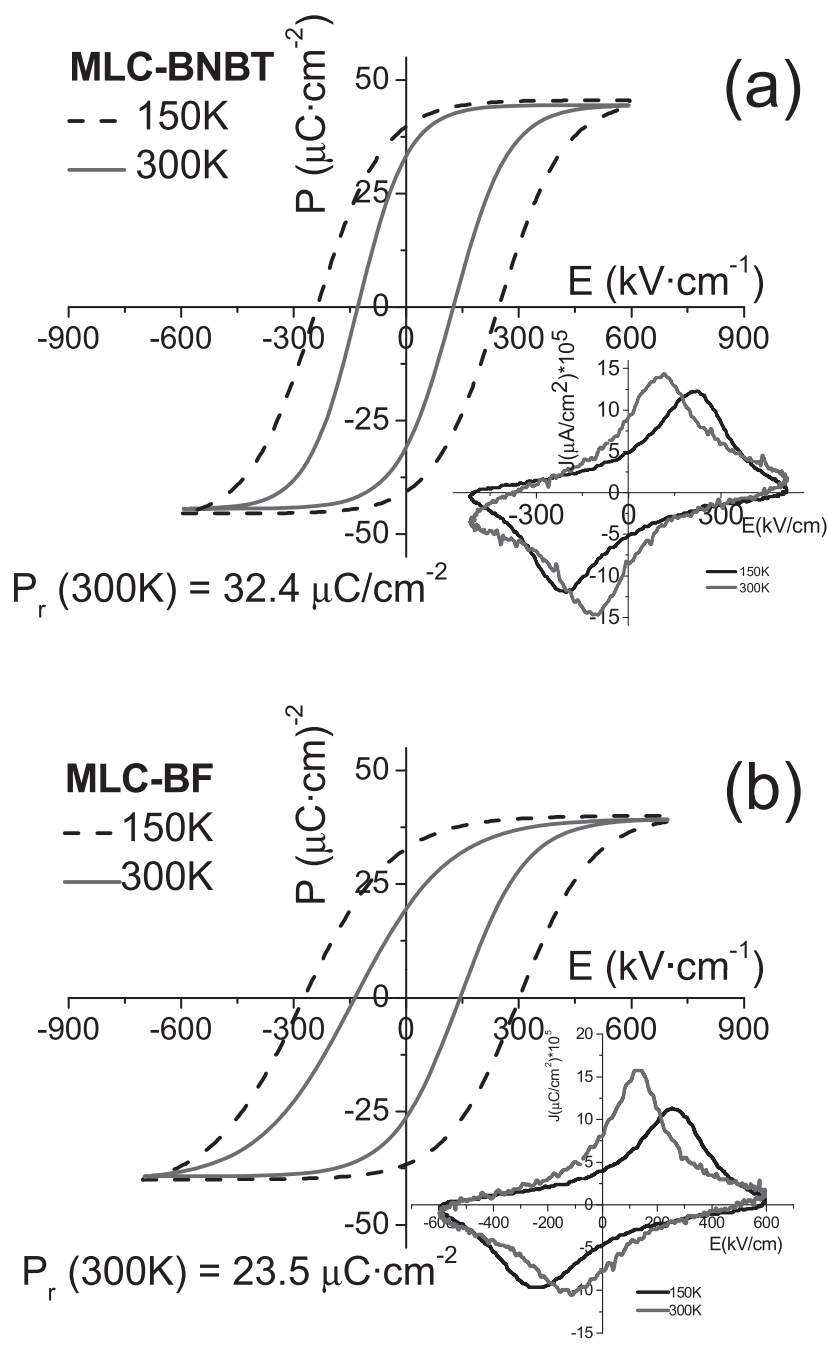

FIG. 3. Corrected P-E hysteresis loops at different temperatures of (a) MLCBNBT and (b) MLC-BF. The insets show the experimental electrical current vs. field loops. experimental results obtained. The parameters to calculate the effective P-E hysteresis loop of this bilayer structure are taken from the loops measured on single phase thin films corresponding to the layers used in the composite. The shape of the loops and the observed depolarization in the single phase films are related to the specific interfacial conditions between the ferroelectric material and the electrode.

We assume the current density continuity through the layers, which leads to the following equation:

$$
\begin{aligned}
J_{B N B T} & =J_{B F}=\sigma_{B N B T} E_{B N B T}+K_{B N B T}^{\prime} \frac{d E_{B N B T}}{d t}+\frac{d P_{B N B T}}{d t} \\
& =\sigma_{B F} E_{B F}+K_{B F}^{\prime} \frac{d E_{B F}}{d t}+\frac{d P_{B F}}{d t}
\end{aligned}
$$

where $\mathrm{J}$ is the current density, $\sigma$ the conductivity, $\mathrm{K}^{\prime}$ the real part of the permittivity, E the electric field, and $\mathrm{P}$ the polarization in each layer. To solve this equation, the method developed by Or et al., ${ }^{25}$ based on the behavior of the polarization with the field proposed by Miller et al., ${ }^{26,27}$ has been used. It must be taken into account that it is important to establish correctly the initial conditions for the first loop: $\mathrm{P}=0$ for $\mathrm{E}=0$ at $\mathrm{t}=0$. The first poling process determines $\mathrm{P}_{\mathrm{s}}$, which will be the same for the following loops. This is especially important in the case of multilayer configurations, as the initial conditions of poling of the different layers are difficult to know a priori. From the calculations, a P-E loop is obtained, which, after subtraction of the non-switching contributions as explained before for the experimental loops, ${ }^{22}$ yields the effective ferroelectric switching P-E loop, comparable with the corrected experimental results (see Figure 4). For MLC-BF, the calculated loop shows a good agreement with the experimental results at room temperature (Figure 4(b)) using for the layers the parameters measured for single phase $\mathrm{BNBT}^{13}$ and $\mathrm{BF}^{18}$ films, collected in Table I. It can be observed that the slope of the loop in the positive branch is almost the same, and the coercive field values are close. The differences observed may be related to small deviations between the single phase film values used and the actual values of the layers forming the composite. However, for 

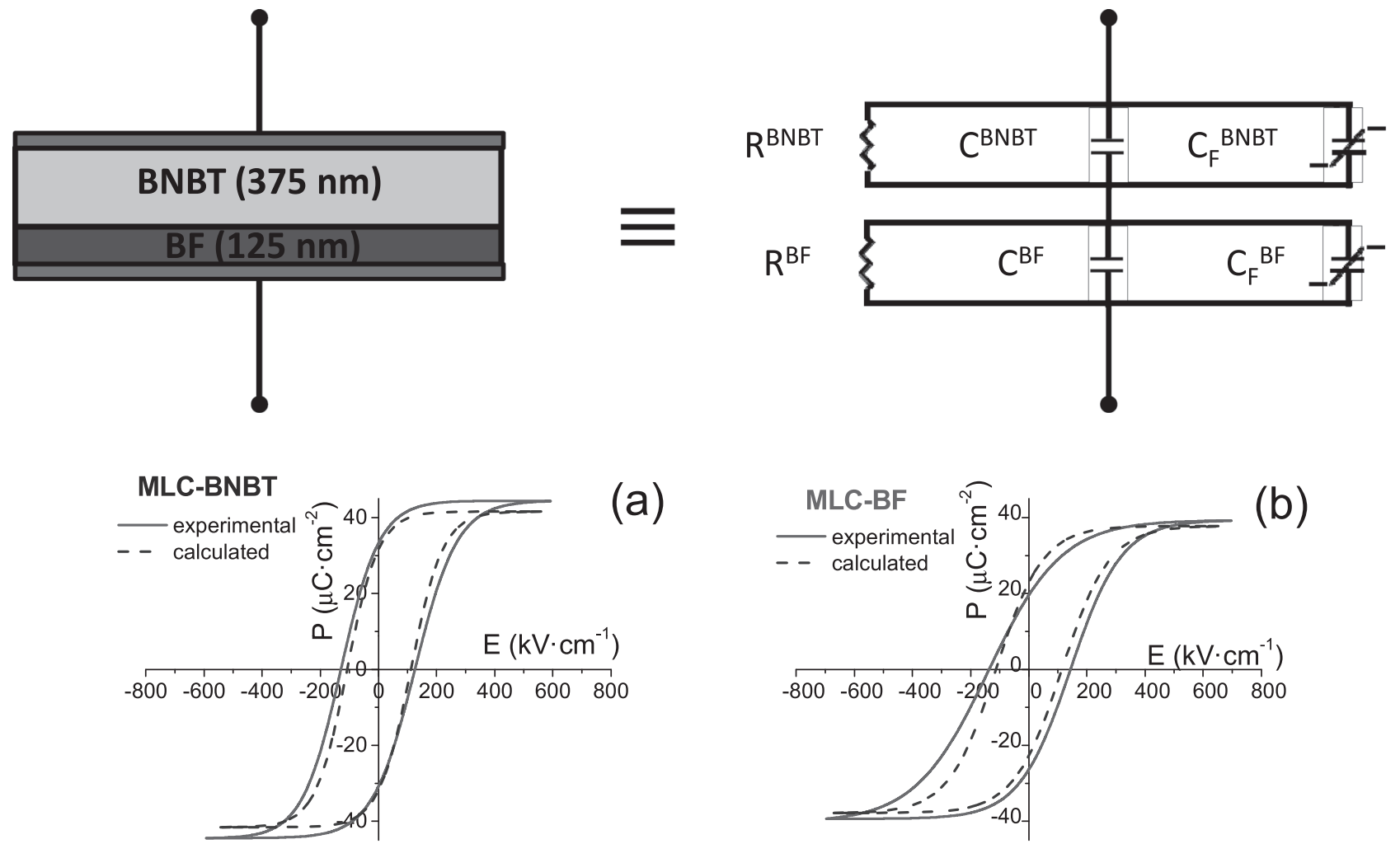

(a)

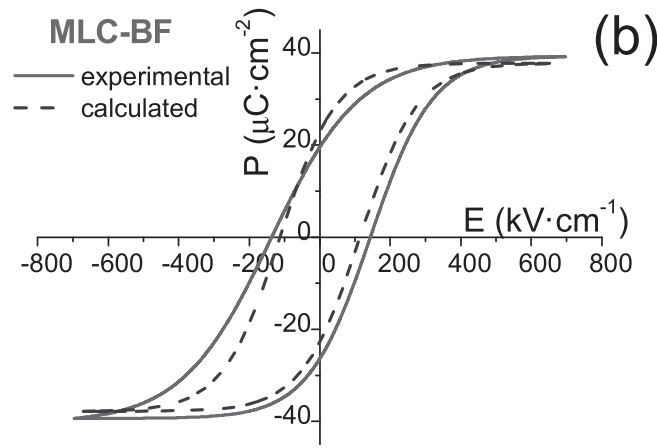

FIG. 4. Bilayer model and equivalent circuit used for the calculation of effective properties of MLC films, and comparison between experimental and calculated P-E loops: (a) MLC-BNBT and (b) MLC-BF.

MLC-BNBT, not only both the $\mathrm{P}_{\mathrm{r}}$ and $\mathrm{P}_{\mathrm{s}}$ values are quite different but also the slope of the loop close to $E_{c}$. This can only be attributed to a significant deviation of the parameters used in the calculations and the real ones. In fact, the calculated loop only fits the experimental data when the input parameters for the polarization of the BNBT layer are increased up to $\mathrm{P}_{\mathrm{s}}=40 \mu \mathrm{C} \times \mathrm{cm}^{-2}$ and $\mathrm{P}_{\mathrm{r}}=25 \mu \mathrm{C} \times \mathrm{cm}^{-2}$, larger than those of the corresponding single phase film (Figure 4(a)). It should be borne in mind that we do not try to fit the experimental loops, but just to obtain a qualitative agreement. This shows that for this MLC film there is an extra contribution to the polarization. This contribution can be attributed to the fact that top and bottom poled BF layers seems to prevent the fast depolarization of the BNBT layer in this configuration, and thus, results in an enhancement of the polarization. It seems that, as expected, ${ }^{6}$ the ordering sequence of the layers in the multilayer composite structure has an influence on the effective P-E loop: it is more square shaped (remnant) when the "hard" layers (BF) are in contact with the electrodes. This layer ordering effect can be related to the depolarization fields developed in the MLC after poling. It is important to stress that we are dealing with fast depolarization phenomena, which means that the depolarization happens between the switching time of the ferroelectric domains (ns) and close to $0.1 \mathrm{~s}$, visible in the time scale of a loop traced at $1 \mathrm{kHz} .{ }^{28}$ One of the main factors of development of depolarization fields $\left(E_{\mathrm{dep}}\right)$ is related to the existence of dead layers of different nature in the interface between the metal electrode and the ferroelectric material $(\mathrm{ME} / \mathrm{FE})$. In the particular case of the occurrence of a non-ferroelectric dead layer of $2 \mathrm{~nm}$ at the ME/FE interface with $\mathrm{K}^{\prime}=40$ for a PZT material with $\mathrm{P}_{\mathrm{s}}$ close to $30 \mu \mathrm{C} \times \mathrm{cm}^{-2}$ and a depolarization field close to $85 \mathrm{kV} \times \mathrm{cm}^{-1}$ can be developed. ${ }^{28}$ In the case of the MLC films, $\mathrm{E}_{\mathrm{dep}}$ in the $\mathrm{ME} / \mathrm{FE}$ interface will be mainly related to the effective saturation polarization obtained. This is larger than the saturation polarization of the single phase BNBT film, and thus the $\mathrm{E}_{\mathrm{dep}}$ (assuming the same interfacial conditions) can be also larger. Interfaces between both ferroelectric layers $(\mathrm{FE} / \mathrm{FE})$ in the MLC films may also be the origin of the depolarization fields. However, the ferroelectric polarization of the layer compensates most of the depolarization field $\mathrm{E}_{\mathrm{dep}}$ produced and, therefore, the role played by these interfaces is less significant. Moreover, if the polarization in the BF layer after poling is higher than that in the BNBT layer, this difference will produce an accumulation of charge

TABLE I. Parameters used in the calculation of the effective ferroelectric properties of MLC films.

\begin{tabular}{lcrrr}
\hline \hline & $\mathrm{P}_{\mathrm{s}}\left(\mu \mathrm{C} \times \mathrm{cm}^{-2}\right)$ & $\mathrm{P}_{\mathrm{r}}\left(\mu \mathrm{C} \times \mathrm{cm}^{-2}\right)$ & $\mathrm{E}_{\mathrm{c}}\left(\mathrm{kV} \times \mathrm{cm}^{-1}\right)$ & $\mathrm{K}^{\prime}$ \\
\hline BF layer $^{18}$ & 65 & 61 & 300 & 400 \\
BNBT layer $^{13}$ (MLC-BF) & 28 & 12 & 68 & 700 \\
BNBT layer [modified] (MLC-BNBT) & 40 & 25 & $7 \mathrm{at}^{-7}$ & 700 \\
\hline \hline
\end{tabular}


in the interface between these layers. This will lead to the stabilization of the BNBT ferroelectric domains reducing the rate of domain reversal in this layer, which can be considered as an effective inducement of additional remnant polarization in the multilayer composite.

We can assume that for the same volume fraction of layers the effective saturation polarization $\mathrm{P}_{\mathrm{s}}$ is similar, and so is the depolarization field $\mathrm{E}_{\text {dep }}$ developed. If the BNBT layer is at the metal/ferroelectric interface (ME/FE), $\mathrm{E}_{\mathrm{dep}}$ can be even larger than its coercive field $\mathrm{E}_{\mathrm{c}}$, and thus it will result in a fast back-switching of domains in this layer, as it happens in the pure single-phase BNBT film. In contrast, if $\mathrm{BF}$ is at the ME/FE interface, the same value of $E_{\mathrm{dep}}$ will produce much slower back-switching due to the high $\mathrm{E}_{\mathrm{c}}$ of the $\mathrm{BF}$, leading to improved remnant properties of the multilayer composite film.

It is also important that the polarization in each layer reaches the saturation values in order to prevent easy backswitching. This can be analyzed through the calculation of the values of the electric field in both layers $\left(\mathrm{E}_{\mathrm{BNBT}}\right.$ and $\left.\mathrm{E}_{\mathrm{BF}}\right)$ using the bilayer model proposed. If a sinusoidal electric field is applied to the MLC films, the model gives the distribution of the electric field in each layer and their evolution with time that are shown in Figure 5(a). While $E_{B N B T}$ follows closely the sinusoidal shape of the applied electric field with a small phase difference, the field in the BF layer, $\mathrm{E}_{\mathrm{BF}}$, shows a differentiated behavior. The shape of the electric field in the BF layer is complex showing the difficulty of
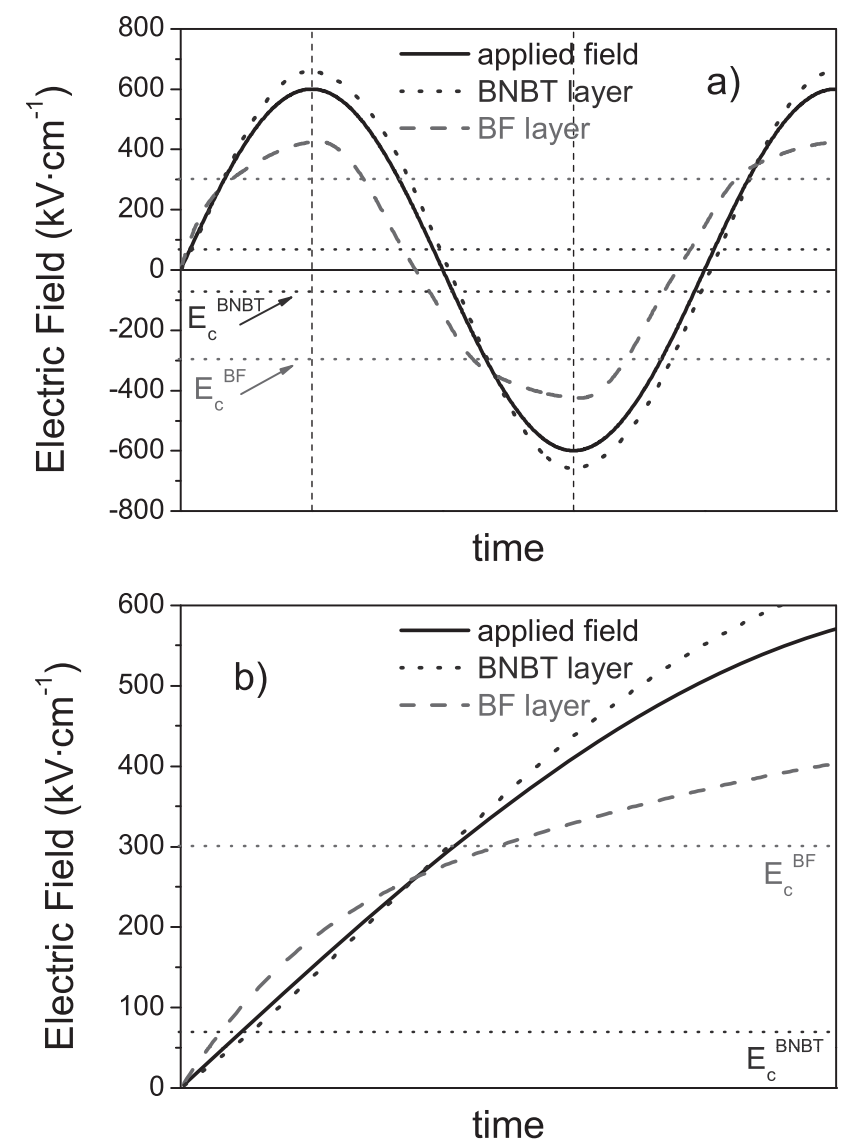

FIG. 5. Calculated electric fields of a bilayer based on the parameters of Table I. The frequency of the applied external field is $1 \mathrm{kHz}$. the ferroelectric switching of the BF in the MLC. The fact that the maximum electric field attained in the BF layer is only $\sim 1.4$ times the coercive field (marked in the graph) results in a calculated $\mathrm{P}_{\mathrm{S}}{ }^{\mathrm{BF}}=57 \mu \mathrm{C} \times \mathrm{cm}^{-2}$, which is below the expected saturation value of $65 \mu \mathrm{C} \times \mathrm{cm}^{-2}$ used for the calculation, see Table I. To reach saturation of the polarization in the BF layer, the electric field applied to the MLC must be higher than $1 \mathrm{MV} \times \mathrm{cm}^{-1}$, which is not possible without the dielectric breakdown of the film. It must be pointed out that this is determined by the difference in permittivity and conductivity between both layers. This under-saturation condition does not happen for the BNBT layer, whose maximum field in the composite is $\sim 10$ times the coercive field (also marked in the graph). Therefore, the choice of the layers of a BNBT-based multilayer must contemplate not only a composition with large polarization and similar conductivity but it is also important that the "hard" layer presents permittivity values lower than the "soft" layer, which will allow an effective polarization of the "hard" layer, reaching saturation values.

It can be observed that the curve of the electric field of the BF layer changes significantly close to the coercive field of the BF layer (Figure 5(b)). This is attributed to the appearance of large currents associated to the polarization switching. For low applied electric fields, the situation is dominated by the switching currents of the BNBT layer, with a lower coercive field (marked in the graph) and so the field is higher in the BF layer. When the applied electric field increases and switching currents start to appear in the BF layer, the balance of the density currents in the MLC changes. At this point, the electric field in the BF layer is smaller and increases at a lower rate than the one in the BNBT layers. The curve changes its slope, as it can be seen in Figure 5(b). It must also be taken into account that the values of the electric field in each layer will be more dissimilar the larger the differences in their switching currents. These depend on both the polarization values and the coercive fields of each layer. In our case, the large difference in the maximum polarization values of the two layers lead to a highly inhomogeneous distribution of the electric fields inside the MLC films. This makes impossible to reach saturation in the BF layers. As a result, a faster depolarization in the $\mathrm{BF}$ layers and consequently at the ferroelectricferroelectric interfaces (FE/FE) may occur. If we do calculations of a similar MLC film, but with layers of similar $\mathrm{P}_{\mathrm{s}}$, like $\mathrm{PbTiO}_{3}$ and $0.65 \mathrm{~Pb}\left(\mathrm{Mg}_{1 / 3} \mathrm{Nb}_{2 / 3}\right) \mathrm{O}_{3}-0.35 \mathrm{PbTiO}_{3}{ }^{6}$ we observe that saturation of the polarization is attained in the two layers. As a result, the effective inducement of stable polarization by reducing the depolarization in one of the layers by the neighboring ones is more efficient. Therefore, in the design of this type of MLC films must be considered the choice of layers with the closest saturation values of the polarization with the aim of getting an improvement in the remnant polarization of the material.

Due to the complex relaxor-ferroelectric nature of the BNBT phase, we must take into account the dependence with the temperature of the polarization of the BNBT layers, ${ }^{13}$ which must affect strongly the remnant polarization values of the MLC films. The values go from $42 \mu \mathrm{C} \times \mathrm{cm}^{-2}$ at $150 \mathrm{~K}$ to 
$32 \mu \mathrm{C} \times \mathrm{cm}^{-2}$ at $300 \mathrm{~K}$ for MLC-BNBT, and from 35 to $23 \mu \mathrm{C} \times \mathrm{cm}^{-2}$ for MLC-BF. This is accompanied by a variation in the coercive field. A study of the variation of the coercive field with temperature is shown in Figure 6(a). The decrease of the coercive field with the increase of temperature in all films can be attributed to the relaxor character of the BNBT layers, which makes polarization unstable with temperature. Figure 6(a) includes the calculations using the bilayer model. In this case, we assume that the parameters of the BF layers do not change much in this range of temperatures (far from its transition temperature). For the BNBT layers, we use the reported values of the single phase films at different temperatures. ${ }^{13}$ There is agreement between experimental and calculated values for the coercive fields at 200 and $300 \mathrm{~K}$, but measured values at $150 \mathrm{~K}$ are larger than expected. The bilayer model cannot reproduce the experimental loop at $150 \mathrm{~K}$ using in the calculation those parameters of the singlephase thin films at the same temperature. The quite strong increase in the effective coercive field can only be reproduced in the bilayer by increasing the coercive field of the BNBT layer. Moreover, to reproduce the experimental P-E loop, it is necessary to increase both $\mathrm{P}_{\mathrm{s}}$ and $\mathrm{P}_{\mathrm{r}}$ simulating a "harder" BNBT layer $\left(\mathrm{P}_{\mathrm{r}}=40 \mu \mathrm{C} \times \mathrm{cm}^{-2} ; \mathrm{P}_{\mathrm{s}}=47 \mu \mathrm{C} \times \mathrm{cm}^{-2}\right.$; and $\left.\mathrm{E}_{\mathrm{C}}=235 \mathrm{kV} \times \mathrm{cm}^{-1}\right)$. The experimental loop and the bilayer calculated loop are plotted together in Figure 7(a). A quite good agreement without performing mathematical fitting is
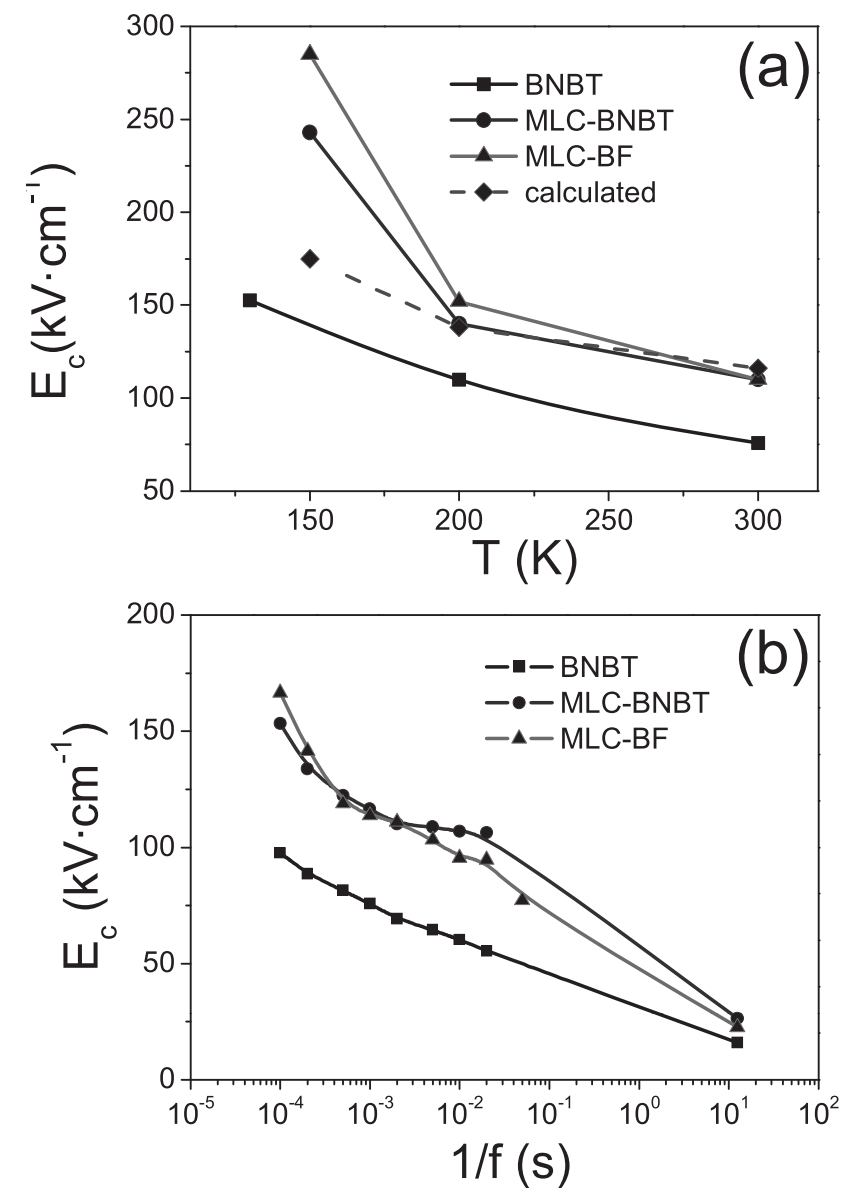

FIG. 6. Evolution of the coercive field EC with (a) temperature and (b) measurement frequency at room temperature for single phase BNBT and MLC films.
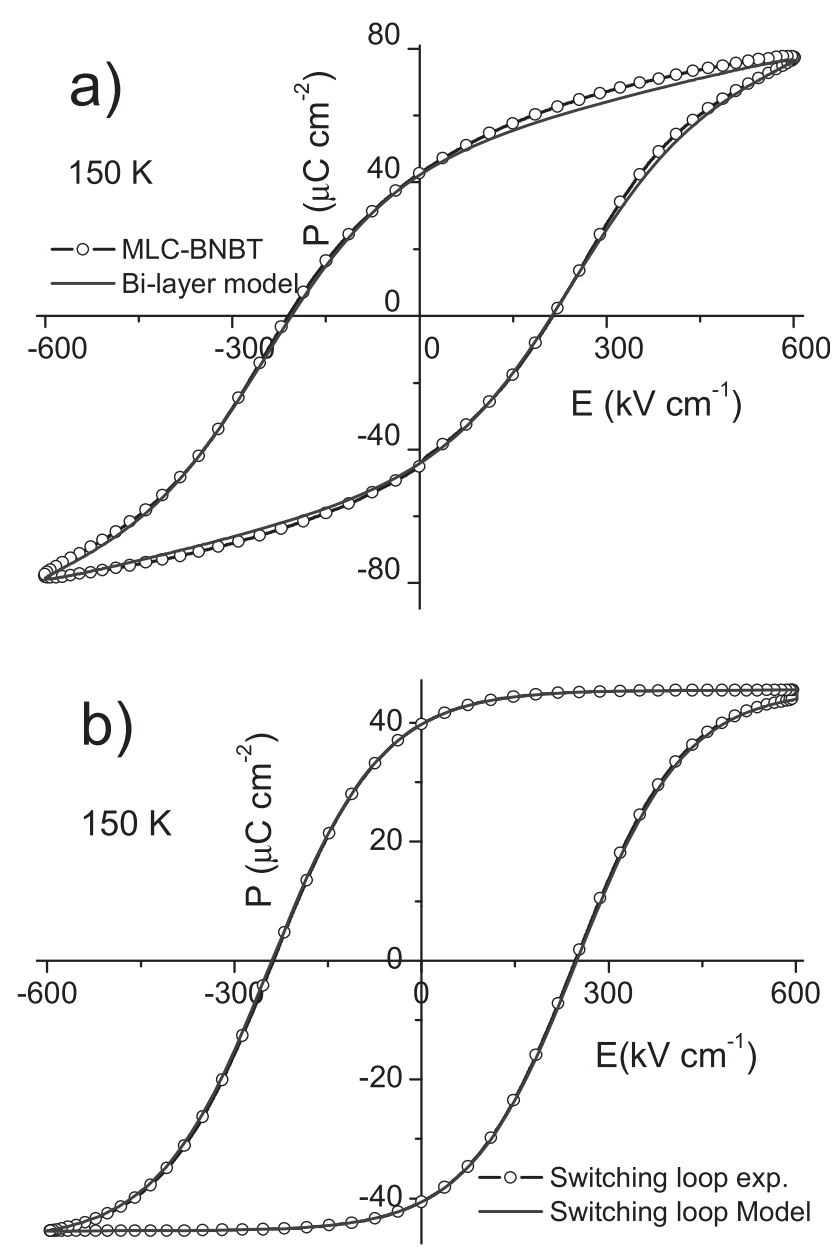

FIG. 7. (a) Experimental (-O-) and calculated (-) P-E hysteresis loops for the MLC-BNBT film. (b) Corrected (-O-) and calculated (-) P-E hysteresis loops for the MLC-BNBT film $(1 \mathrm{kHz}, 150 \mathrm{~K})$.

obtained. In Figure 7(b), the experimental corrected ferroelectric switching loop and the calculated one are included, showing almost a perfect agreement. It is important to stress that the calculated loop is very sensitive to small changes in the parameters used for the layers. This result is an evidence of the higher stability of the polarization in the BNBT layers at the lowest temperatures. It can be argued that the BNBT layers can develop larger and more stable ferroelectric domains after poling with the large electric fields produced in this layer at low temperatures. It should be pointed out that the field in the BNBT layer is quite high in the MLC structure, see Figure 5 (10 times $\mathrm{E}_{\mathrm{c}}$ ). Besides, this agrees with reports on the BNBT bulk ceramics which presents quite squareshaped loops with high coercive fields at room temperature. ${ }^{11}$ On the other hand, and regarding the complex relaxor behavior of this compound, $150 \mathrm{~K}$ is quite far from the experimental freezing temperature $T_{f}$ obtained in thin films: $408 \mathrm{~K} .{ }^{13}$ Therefore, poling at this temperature can produce more stable ferroelectric domains that should lead to an increase of the $P_{s}$ and the $\mathrm{P}_{\mathrm{r}}$ as observed here. In a recent paper, it has experimentally been demonstrated that in BNBT close to the morphotropic phase boundary; it is possible to attain $\mathrm{P}_{\mathrm{s}}$ values in excess of $40 \mu \mathrm{C} \times \mathrm{cm}^{-2} .{ }^{29}$ Thus, it is plausible to expect an increase in the ferroelectric parameters of BNBT films under intense fields and far from $\mathrm{T}_{\mathrm{f}}$. 
If an enhancement of the ferroelectric properties of the BNBT layer is produced, they will become closer to those of the BF layer, and therefore it will allow a more effective poling of the BF layer as discussed before. As a result, the effective inducement of polarization sought in these MLC configurations will be more efficient and better retention properties are found at low temperatures.

As a consequence, we can say that on approaching room temperature (closer to $\mathrm{T}_{\mathrm{f}}$ ), the induced ferroelectric domains in the BNBT layers are less and less stable. The increasing instability of these relaxor layers produces strong changes of the coercive fields with the measurement frequency as observed at room temperature (Figure 6(b)). Values of $E_{c}$ are obtained from P-E loops measured at different frequencies. At the lowest frequency, the static coercive field from the C$\mathrm{V}$ loops is considered. We can see that the coercive field decreases with the inverse of the frequency for all films. From $10^{-4}$ to $10^{-3}$ seconds, the decrease is fast and can be related to the depolarization effects related to the large screening length in the electrodes. ${ }^{30}$ For lower frequencies, the MLC-BNBT films present a slight reduction till $0.02 \mathrm{~s}$. Differently, the MLC-BF film presents a larger reduction indicating larger ferroelectric instability phenomena in this MLC. Finally, for the static $E_{c}$ there is a strong reduction in the values that can be related to the long term depolarization of the BNBT and BF layers. This behavior is typical of relaxor-ferroelectric materials and indicates that none of the two configurations used in the MLC films with the BF layers does alter the relaxor-like character of the BNBT layers at room temperature.

For applications, it is important to check the behavior of the remnant polarization with time. The retention of the polarization at room temperature and after $1 \mathrm{~s}$ of relaxation in both MLCs was studied through remnant hysteresis loops (Figure 8(a)), where the values are measured $1 \mathrm{~s}$ after the application of the electric field. It can be seen that both MLCs present values of $\mathrm{P}_{\mathrm{r}}$ below those obtained for the P-E loops of Figure 3: $\mathrm{P}_{\mathrm{r}}=15.8 \mu \mathrm{C} \times \mathrm{cm}^{-2}$ for MLC-BNBT $\mathrm{P}_{\mathrm{r}}=12.7 \mu \mathrm{C} \times \mathrm{cm}^{-2}$ for MLC-BF. This indicates a relaxation of $\sim 50 \%$ in $1 \mathrm{~s}$, which is much less than the one reported for BNBT single phase film: $\sim 75 \%{ }^{13}$ Besides, the effective induced polarization in the MLC-BNBT must be still present, as its $\mathrm{P}_{\mathrm{r}}$ is still larger than that of MLC-BF, although it has also been reduced to $24 \%$. This must be compared with $33 \%$ obtained in the P-E loops, where the measurement is done with the electric field applied. In a further study of the retention of this polarization for longer times (Figure 8(b)), we observe that the values are rather stable after the first few seconds, when an additional $15 \%-20 \%$ of the polarization is lost. It has been observed that after around $3 \mathrm{~h}$ from poling the values of the remnant polarization remain around $15 \mu \mathrm{C} \times \mathrm{cm}^{-2}$. All of this indicates that, after a quick relaxation of the polarization for very short times, most probably related to the polarization instability after the removal of the electric field in the relaxor-like state of the BNBT layers, some volume of oriented ferroelectric domains remains in the composites and this volume is rather stable with time.

In order to apply large electric fields, the appearance of leakage currents must be considered. This has been
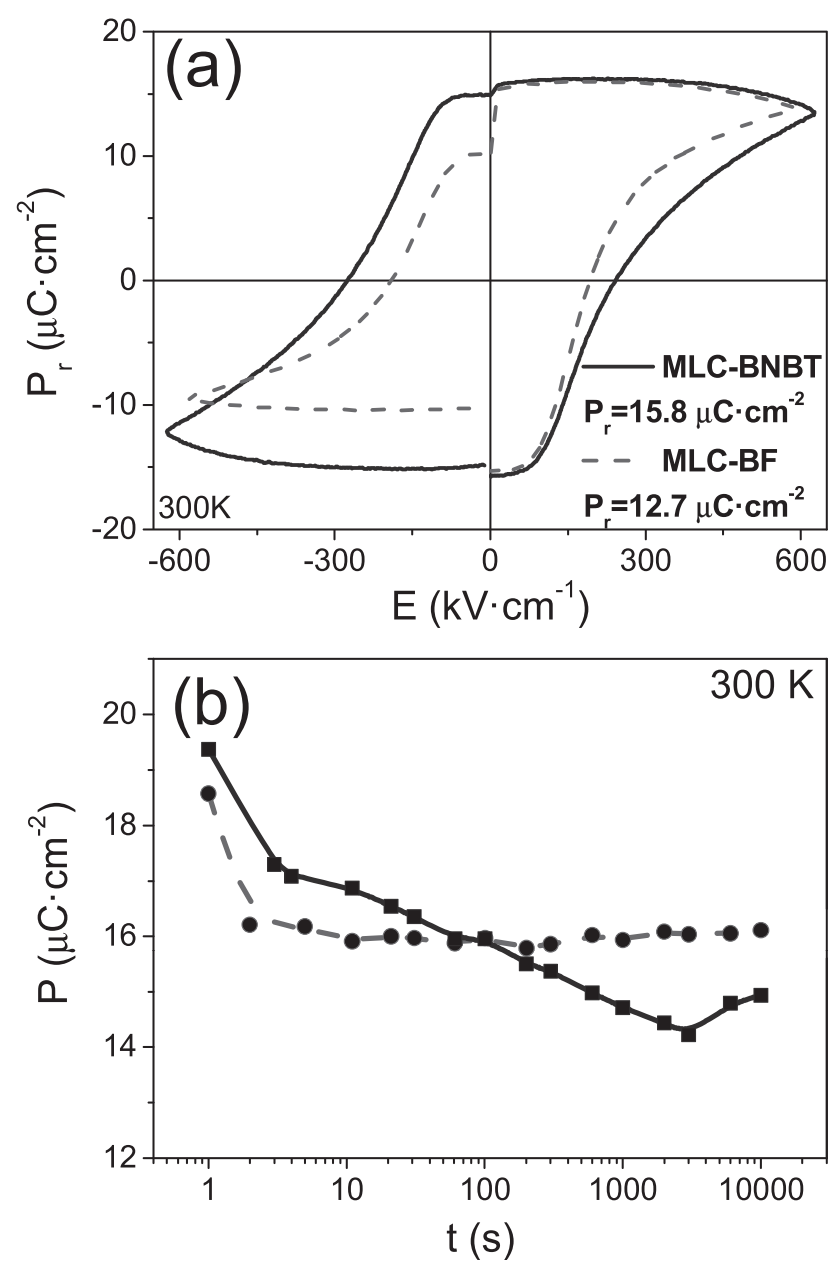

FIG. 8. (a) Remnant hysteresis loops of multilayer composite films. (b) Measurement of the retention of the polarization for longer times.

traditionally a problem for BF films, as it can be seen in Figure 9, where a comparison of the behavior of the current density vs. electric field are compared for the single phase and multilayer composite films. Single phase BF films present values of current density in the range of $10^{-7}$ to $10^{-5} \mathrm{~A} \times \mathrm{cm}^{-2}$ when we first apply an electric field. However, when we increase the values of the applied electric field above approximately $140 \mathrm{kV} \times \mathrm{cm}^{-1}$, the film suffers a degradation and the conductivity values goes up to $0.1-1 \mathrm{~A} \times \mathrm{cm}^{-2}$. Although the reasons of this behavior are still not clear, it seems to be related with the point defects, their relation with the extended defects and the large FE polarization in the $\mathrm{BF} .{ }^{17}$ As commented previously this behavior can be prevented or attenuated by the series combination with other FE layers that can modulate the field in the $\mathrm{BF}$ layer and act as buffer layers for the point defects as, for example, the BNBT. As can be observed in Figure 9, the single phase film of BNBT presents low values of the leakage current and no degradation. Therefore, both MLC films present intermediate current density values between those of pure BNBT and BF films and no degradation phenomena for large electric fields. The most effective layer combination from this point of view seems to be that of MLC-BF, where the BF layer is sandwiched between two BNBT layers $\left(10^{-8}\right.$ $\mathrm{A} \times \mathrm{cm}^{-2}$ at $150 \mathrm{kV} \times \mathrm{cm}^{-1}$ ). In this case, there is not 


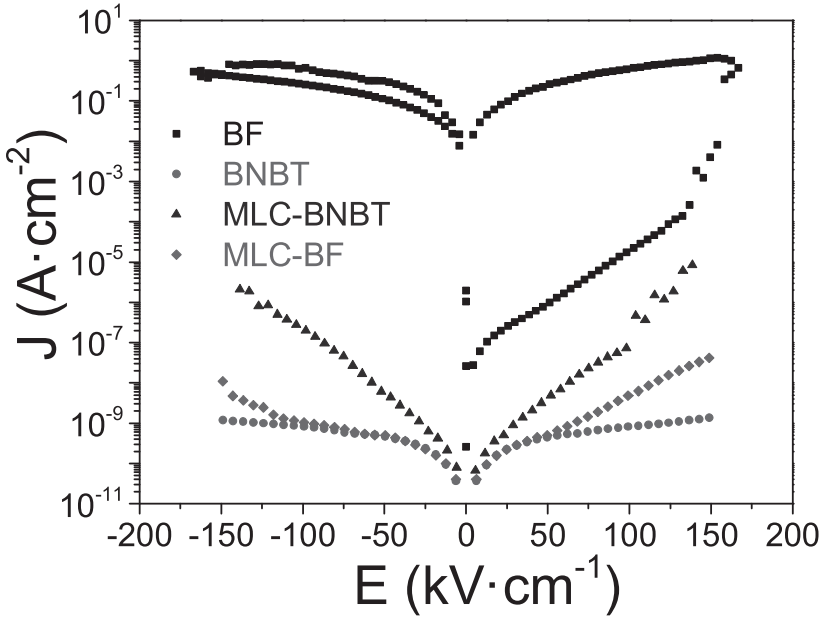

FIG. 9. Leakage currents of the single phase $\mathrm{BiFeO}_{3}$ and BNBT films compared with the multilayer composite films MLC-BNBT and MLC-BF.

interface of the BF layers with the electrode. The control of the leakage currents in BF-based films is essential for their application in magnetoelectric devices.

Figure 10 shows the M-H hysteresis loops of both MLC films. It can be seen that both show a magnetic behavior with neat remanence and coercivity, as it is shown in the insets of
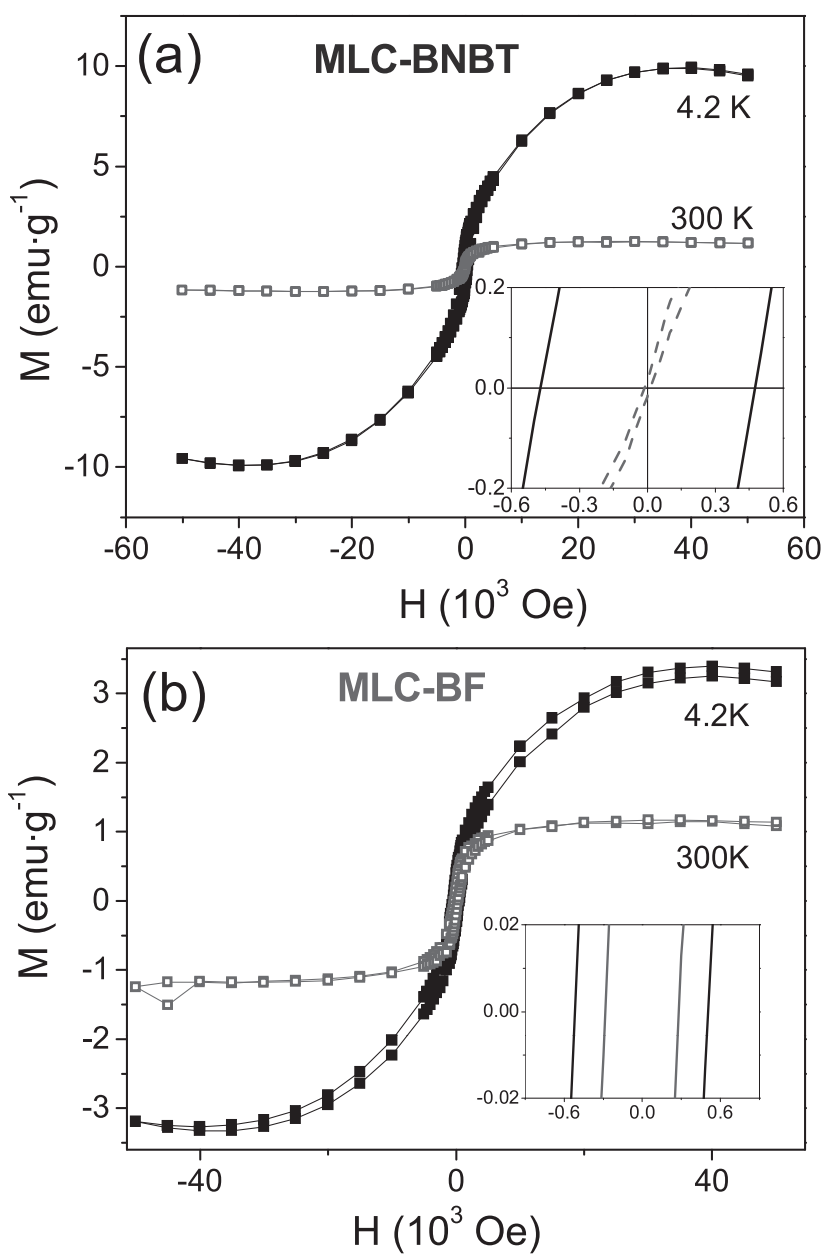

FIG. 10. M-H hysteresis loops at different temperatures of (a) MLC-BNBT and (b) MLC-BF films. The insets show a magnified view of the central region of the loops. the figure. This is not the expected behavior for the magnetic BF layers. It has been reported that the antiferromagnetic spin order of $\mathrm{BiFeO}_{3}$ is not homogenous, but space modulated, ${ }^{31}$ as an incommensurate cycloid structure. The antiferromagnetic vector is normally locked within the cycloid, averaged to zero, but the effect of an epitaxial constraint in a film can release this latent magnetization. ${ }^{32}$ However, the same effect has been observed for polycrystalline BF films with columnar grain growth ${ }^{18}$ similar to the MLC films studied here. We can argue that the origin of the enhanced magnetization in these films can be attributed to strain effects on the columnar grains, in analogy with the epitaxial films but finite size effects may be also responsible for the appearance of a neat magnetization. Similar to them, we can also find a correlation between thickness and saturation magnetization as reported for epitaxial BF films. ${ }^{16}$ The MLC-BF presents values of the saturation magnetization: $\mathrm{M}_{\mathrm{S}} \sim 3.3 \mathrm{emu} \times \mathrm{g}^{-1}$ at $4.2 \mathrm{~K}$ and $1.1 \mathrm{emu}$ $\times \mathrm{g}^{-1}$ at room temperature with an estimated thickness for the BF layer of $\sim 120 \mathrm{~nm}$ (estimated from the number of solution depositions used). These values increase in the case of MLC-BNBT up to $\mathrm{M}_{\mathrm{S}} \sim 9.8 \mathrm{emu} \times \mathrm{g}^{-1}$ at $4.2 \mathrm{~K}$ and $\sim 1.3$ $\mathrm{emu} \times \mathrm{g}^{-1}$ at room temperature, where the thickness of the $\mathrm{BF}$ layers are reduced to around $\sim 60 \mathrm{~nm}$. The comparison of the XRD peak positions obtained for the films (Figures 1(b) and 1(c)) with those for ceramic powders [JCPDS 86-1518], indicate a slight shift of the BF peaks to larger angles in both MLCs that can be related to a similar reduction of the cell parameters in the BF layers. On the contrary, the position of the BNBT peak in both MLCs is very close to that of the ceramic powder Ref. 24 indicating that the BNBT layers are almost relaxed.

Therefore, we can conclude that finite size effects are dominant and that if we are searching for larger values of the magnetization in these films, despite stress effects on the observed magnetization cannot be discarded, BF layers need to be as thin as possible.

\section{CONCLUSIONS}

MLC films of $\left(\mathrm{Bi}_{0.5} \mathrm{Na}_{0.5}\right)_{0.945} \mathrm{Ba}_{0.055} \mathrm{TiO}_{3}$ and $\mathrm{BiFeO}_{3}$ have been prepared by chemical solution deposition, achieving the desired phases and without appreciable interdiffusion between the different layers. The combination of these layers results in the improvement of the remnant polarization with respect to the values obtained for pure BNBT films through the effective preservation of the remnant polarization in the BNBT layer by surrounding poled BF layers.

It can be deduced from the results obtained that the best properties for these multilayer composite films will be obtained when the layers have similar saturation values of the polarization. Not only that, those layers in contact with the electrode should have large enough coercive fields. The aim is always to hinder the effects of the depolarization fields, which leads to the stabilization of the long range ferroelectric order in the layers. As a consequence an effective inducement of added remnant polarization is obtained.

Besides, the presence of the BNBT layers leads to the reduction of the leakage currents at room temperature in the MLC films. These are a strong problem for the use of the 
multiferroic BF thin films at room temperature in applications. The magnetic behavior at room temperature of the MLC-BNBT films shows the rupture of the antiferromagnetic cycloid and the appearance of an enhanced remnant magnetization. This, together with the stable remnant polarization obtained, make these multilayer composite thin films very promising for their integration in room temperature multiferroic devices.

\section{ACKNOWLEDGMENTS}

This work has been funded by the Spanish Project MAT2010-15365, MAT2013-40489-P, and MAT2011-C0202. A. Perez-Rivero acknowledges the funding of his Spanish FPI Ph.D. grant. Dr. I. Bretos is a postdoctoral fellow of the Spanish Juan de la Cierva programme.

${ }^{1}$ N. Setter, D. Damjanovic, L. Eng, G. Fox, S. Gevorgian, S. Hong, A. Kingon, H. Kohlstedt, N. Y. Park, G. B. Stephenson, I. Stolitchnov, A. K. Taganstev, D. V. Taylor, T. Yamada, and S. Streiffer, J. Appl. Phys. 100, 051606 (2006).

${ }^{2}$ D. Bao, Curr. Opin. Solid State Mater. Sci. 12, 55 (2008).

${ }^{3}$ H. N. Lee, H. M. Christen, M. F. Chisholm, C. M. Rouleau, and D. H. Lowndes, Nature 433, 395-399 (2005).

${ }^{4}$ F. M. Pontes, E. Longo, E. R. Leite, and J. A. Varela, Appl. Phys. Lett. 84, 5470-5472 (2004).

${ }^{5}$ M. Algueró, M. Stewart, M. G. Cain, P. Ramos, J. Ricote, and M. L. Calzada, J. Phys. D: Appl. Phys. 43, 205401 (2010).

${ }^{6} \mathrm{H}$. El Hosiny Ali, R. Jiménez, J. Ricote, J. Pérez de la Cruz, J. R. A. Fernandes, and M. L. Calzada, Thin Solid Films 520, 7205-7211 (2012).

${ }^{7}$ R. Guo, L. E. Cross, S. E. Park, B. Noheda, D. E. Cox, and G. Shirane, Phys. Rev. Lett. 84, 5423-5426 (2000).

${ }^{8}$ J. Rödel, W. Jo, K. T. P. Seifert, E.-M. Anton, T. Granzow, and D. Damjanovic, J. Am. Ceram. Soc. 92, 1153-1177 (2009).

${ }^{9}$ T. Takenaka, K. Maruyama, and K. Sakata, Jpn. J. Appl. Phys., Part 1 30, 2236-2239 (1991).

${ }^{10}$ Y.-M. Chiang, G. W. Farrey, and A. N. Soukhojak, Appl. Phys. Lett. 73, 3683-3685 (1998).

${ }^{11}$ W. Jo, S. Silke, E. Sapper, L. A. Schmitt, H. J. Kleebe, A. J. Bell, and J. Rödel, J. Appl. Phys. 110, 074106 (2011).
${ }^{12}$ D. Pérez-Mezcua, R. Sirera, I. Bretos, J. Ricote, R. Jiménez, L. FuentesCobas, R. Escobar-Galindo, D. Chateigner, and M. L. Calzada, J. Am. Ceram. Soc. 97, 1269-1275 (2014).

${ }^{13}$ A. Perez-Rivero, J. Ricote, I. Bretos, M. L. Calzada, J. Pérez de la Cruz, J. R. A. Fernandes, and R. Jiménez, J. Am. Ceram. Soc. 97, 1276-1282 (2014).

${ }^{14}$ M. Algueró, J. Ricote, R. Jiménez, P. Ramos, J. Carreaud, B. Dkhil, J. M. Kiat, J. Holc, and M. Kosec, Appl. Phys. Lett. 91, 112905 (2007).

${ }^{15}$ G. Catalan and J. F. Scott, Adv. Mater. 21, 2463-2485 (2009).

${ }^{16}$ J. Wang, J. B. Neaton, H. Zheng, V. Nagarajan, S. B. Ogale, B. Liu, D. Viehland, V. Vaithyanathan, D. G. Schlom, U. V. Waghmare, N. A. Spaldin, K. M. Rabe, M. Wuttig, and R. Ramesh, Science 299, 1719 (2003).

${ }^{17}$ I. Bretos, R. Jiménez, C. Gutiérrez-Lázaro, I. Montero, and M. L. Calzada, Appl. Phys. Lett. 104, 092905 (2014).

${ }^{18}$ C. Gutiérrez-Lázaro, I. Bretos, R. Jiménez, J. Ricote, H. El Hosiny, D. Pérez-Mezcua, R. J. Jiménez Rioboo, M. García-Hernández, and M. L. Calzada, J. Am. Ceram. Soc. 96(10), 3061-3069 (2013).

${ }^{19}$ S. Hoffmann and R. Waser, J. Eur. Ceram. Soc. 19(6-7), 1339-1343 (1999).

${ }^{20}$ D. Alonso-Sanjosé, R. Jiménez, I. Bretos, and M. L. Calzada, J. Am. Ceram. Soc. 92, 2218-2225 (2009).

${ }^{21}$ D. Rivero, L. Pardo, and R. Jiménez, Rev. Cubana Fís. 26(1A), 169-173 (2009), ISSN:0253-9268.

${ }^{22}$ R. Jiménez, C. Alemany, M. L. Calzada, A. González, J. Ricote, and J. Mendiola, Appl. Phys. A 75, 607-615 (2002).

${ }^{23}$ T. P. Juan, S. Chen, and J. Y. Lee, J. Appl. Phys. 95, 3120 (2004).

${ }^{24}$ D. Pérez-Mezcua, M. L. Calzada, I. Bretos, J. Ricote, D. Chateigner, R. Escobar-Galindo, R. Jiménez, and R. Sirera, Bol. Soc. Esp. Ceram. Vidrio 53, 21-26 (2014).

${ }^{25}$ Y. T. Or, C. K. Wong, B. Ploss, and F. G. Shin, J. Appl. Phys. 93, 4112 (2003).

${ }^{26}$ S. L. Miller, R. D. Nasby, J. R. Schwank, M. S. Rodgers, and P. V. Dressendorfer, J. Appl. Phys. 68, 6463 (1990).

${ }^{27}$ S. L. Miller, J. R. Schwank, R. D. Nasby, and M. S. Rodgers, J. Appl. Phys. 70, 2849 (1991)

${ }^{28}$ X. J. Lou, J. Appl. Phys. 105, 094107 (2009).

${ }^{29}$ D. Schneider, W. Jo, J. Roedel, D. Rytz, and T. Granzow, J. Appl. Phys. 116, 044111 (2014).

${ }^{30}$ M. Dawber, P. Chandra, P. B. Littlewood, and J. F. Scott, J. Phys.: Condens. Matter 15, L393-L398 (2003).

${ }^{31}$ I. Sosnowska, T. Peterlin-Neumaier, and E. Steichele, J. Phys. C: Solid State Phys. 15, 4835 (1982).

${ }^{32}$ F. Bai, J. Wang, M. Wuttig, J. F. Li, N. Wang, A. P. Pyatakov, A. K. Zvezdin, L. E. Cross, and D. Viehland, Appl. Phys. Lett. 86, 032511 (2005). 